\title{
Pre-operative Education for Total Knee Replacement: A Pilot Survey
}

\author{
Renee Causey-Upton \\ Eastern Kentucky University, renee.causey-upton@eku.edu
}

Dana M. Howell

Eastern Kentucky University, dana.howell@eku.edu

Patrick H. Kitzman

University of Kentucky, phkitz1@uky.edu

Melba Custer

Spalding University, Mcuster@spalding.edu

Emily V. Dressler

Wake Forest University, edressle@wakehealth.edu

Follow this and additional works at: https://nsuworks.nova.edu/ijahsp

Part of the Rehabilitation and Therapy Commons

\section{Recommended Citation}

Causey-Upton R, Howell DM, Kitzman PH, Custer M, Dressler EV. Pre-operative Education for Total Knee Replacement: A Pilot Survey. The Internet Journal of Allied Health Sciences and Practice. 2018 Jan 01;16(4), Article 6.

This Manuscript is brought to you for free and open access by the College of Health Care Sciences at NSUWorks. It has been accepted for inclusion in Internet Journal of Allied Health Sciences and Practice by an authorized editor of NSUWorks. For more information, please contact nsuworks@nova.edu. 


\title{
Pre-operative Education for Total Knee Replacement: A Pilot Survey
}

\begin{abstract}
Purpose: The structure of pre-operative education programs used nationally for patients prior to total knee replacement (TKR) surgery has not been identified previously, thus hospitals across the United States lack a common standard for this pre-operative education to ensure best patient outcomes. The purpose of this pilot survey study was to describe the content, providers, and delivery methods currently utilized to deliver pre-operative education for total knee replacement in the United States.

Method: Data were collected using an online survey developed by the authors based on review of literature and three pre-operative programs, and was distributed through the Research Electronic Data Capture (REDCap). The survey consisted of 16 questions, including 12 closed-ended and four open-ended items. Participants were identified through convenience sampling using contacts of the first author and an internet search of hospitals that provide pre-operative education. Seven professional participants total from nursing, physical therapy, and occupational therapy completed the survey. Descriptive statistics were used for data analysis of the 12 quantitative questions to determine frequency and percentages of responses. Responses on the four open-ended survey items, as well as participant responses of "other" for question items, were recorded and collated from individual survey responses.
\end{abstract}

Results: Pre-operative education provider teams for total knee replacements most frequently consisted of nursing, physical therapy, and occupational therapy staff. Most education programs were provided two weeks prior to surgery in a group format, with the majority of programs being delivered in a single session lasting between 1 and 1.5 hours. Verbal and written instruction were the most commonly utilized methods to deliver education. Individual patient programs included a variety of topics, ranging from what to expect while in the hospital, self-care, adaptive equipment, and home safety being some of the most commonly included content.

Conclusions: This pilot study provides a framework to describe the structure of pre-operative total knee replacement education nationally, and can be used to guide a future large scale survey to fully describe the content, providers, and delivery methods of pre-operative education for this population across the United States using a representative sample.

\section{Author Bio(s)}

Renee Causey-Upton, PhD, OTD, OTR/L, has a PhD in Rehabilitation Sciences from University of Kentucky. She is an assistant professor and the Bachelor of Science in Occupational Science Program Coordinator for the Department of Occupational Science and Occupational Therapy at Eastern Kentucky University. Dana M. Howell, PhD, OTD, OTR/L, FAOTA, has a PhD in Education from the University of Idaho and is a full professor in the Department of Occupational Science and Occupational Therapy at Eastern Kentucky University. She is also the Occupational Therapy Doctorate Program Coordinator at Eastern Kentucky University and Rehabilitation Sciences Doctoral program Liaison at University of Kentucky. Patrick H. Kitzman, PT, PhD, has a PhD in Neurosciences from the Ohio State University and is a full professor in the Department of Rehabilitation Sciences at the University of Kentucky. He also serves as the Associate Dean for Research in the College of Health Sciences at the University of Kentucky. Melba Custer, PhD, OT/ $\mathrm{L}$, has a PhD in Rehabilitation Sciences from University of Kentucky and is a professor in the Department of Occupational Therapy at Spalding University. She is also an occupational therapist at Commonwealth Hand Therapy. Emily V. Dressler, PhD, has her PhD in Biostatistics from the Medical University of South Carolina and is currently an associate professor in the Department of Biostatistical Sciences at Wake Forest School of Medicine. 


\section{Acknowledgements}

The authors would like to thank the health care providers who were willing to participate in this pilot survey. 


\section{Preoperative Education for Total Knee Replacement: A Pilot Survey}

Renee Causey-Upton

Dana M. Howell

Patrick H. Kitzman

Melba Custer

Emily V. Dressler

Follow this and additional works at: https://nsuworks.nova.edu/ijahsp Part of the Rehabilitation and Therapy Commons

This Manuscript has supplementary content. View the full record on NSUWorks here: 


\begin{abstract}
Purpose: The structure of preoperative education programs used nationally for patients prior to total knee replacement (TKR) surgery has not been identified previously, thus hospitals across the United States lack a common standard for this preoperative education to ensure best patient outcomes. The purpose of this pilot survey study was to describe the content, providers, and delivery methods currently utilized to deliver preoperative education for total knee replacement in the United States.
\end{abstract}

Method: Data were collected using an online survey developed by the authors based on review of literature and 3 preoperative programs and was distributed through the Research Electronic Data Capture (REDCap). The survey consisted of 16 questions, including 12 closed-ended and 4 open-ended items. Participants were identified through convenience sampling using contacts of the first author and an Internet search of hospitals that provided preoperative education. Seven professional participants from nursing, physical therapy, and occupational therapy completed the survey. Descriptive statistics were used for data analysis of the 12 quantitative questions to determine frequency and percentages of responses. Responses on the 4 open-ended survey items as well as participant responses of "other" for question items were recorded and collated from individual survey responses.

Results: Preoperative education provider teams for total knee replacements most frequently consisted of nursing, physical therapy, and occupational therapy staff. Most education programs were provided 2 weeks prior to surgery in a group format with the majority of programs being delivered in a single session lasting between 1 and 1.5 hours. Verbal and written instruction were the most commonly utilized methods to deliver education. Individual patient programs included a variety of topics, ranging from the expectations while in the hospital, self-care, adaptive equipment, and home safety, being some of the most commonly included content.

Conclusions: With this pilot study, we have provided a framework to describe the structure of preoperative total knee replacement education nationally, which can be used to guide a future large-scale survey to fully describe the content, providers, and delivery methods of preoperative education for this population across the United States, using a representative sample. 


\title{
Preoperative Education for Total Knee Replacement: a Pilot Survey
}

\author{
Renee Causey-Upton, PhD, OTD, OTR/L ${ }^{1}$ \\ Dana M.Howell, PhD, OTD, OTR/L, FAOTA ${ }^{1}$ \\ Patrick H. Kitzman, $\mathrm{PT}, \mathrm{PhD}^{2}$ \\ Melba Custer, PhD, OT/L ${ }^{3}$ \\ Emily V. Dressler, $\mathrm{PhD}^{4}$ \\ 1. Eastern Kentucky University \\ 2. University of Kentucky \\ 3. Kentucky University \\ 4. Wake Forest School of Medicine
}

United States

\begin{abstract}
Purpose: The structure of preoperative education programs used nationally for patients prior to total knee replacement (TKR) surgery has not been identified previously, thus hospitals across the United States lack a common standard for this preoperative education to ensure best patient outcomes. The purpose of this pilot survey study was to describe the content, providers, and delivery methods currently utilized to deliver preoperative education for total knee replacement in the United States. Method: Data were collected using an online survey developed by the authors based on review of literature and 3 preoperative programs and was distributed through the Research Electronic Data Capture (REDCap). The survey consisted of 16 questions, including 12 closed-ended and 4 open-ended items. Participants were identified through convenience sampling using contacts of the first author and an Internet search of hospitals that provided preoperative education. Seven professional participants from nursing, physical therapy, and occupational therapy completed the survey. Descriptive statistics were used for data analysis of the 12 quantitative questions to determine frequency and percentages of responses. Responses on the 4 open- ended survey items as well as participant responses of "other" for question items were recorded and collated from individual survey responses. Results: Preoperative education provider teams for total knee replacements most frequently consisted of nursing, physical therapy, and occupational therapy staff. Most education programs were provided 2 weeks prior to surgery in a group format with the majority of programs being delivered in a single session, lasting between 1 and 1.5 hours. Verbal and written instruction were the most commonly utilized methods to deliver education. Individual patient programs included a variety of topics, ranging from the expectations while in the hospital, self-care, adaptive equipment, and home safety, being some of the most commonly included content. Conclusions: With this pilot study, we have provided a framework to describe the structure of preoperative total knee replacement education nationally, which can be used to guide a future large-scale survey to fully describe the content, providers, and delivery methods of preoperative education for this population across the United States, using a representative sample.
\end{abstract}

Key Words: total knee arthroplasty, total joint replacement, pre-surgical, training 


\section{BACKGROUND}

The prevalence of symptomatic knee osteoarthritis (OA) in the United States in 2016 was estimated to include 14 million persons currently living with knee OA. ${ }^{1}$ Total knee replacement (TKR) is a common intervention for OA that has been found to have better outcomes than non-surgical interventions with greater than 750,000 persons undergoing TKR annually in the United States. ${ }^{2,3}$ TKR has often been associated with reduced pain, increased quality of life, improved functional abilities, and high patient satisfaction following surgery. ${ }^{4}$ Although most persons who undergo TKR experience these positive results, others do not achieve the same satisfactory outcomes. Reduced functional performance, falling in hospital or at home, hospital re-admittance, and other negative outcomes have been reported in the literature following TKR surgery. The functional limitations experienced in the early phase after TKR have often been greater than patients anticipated with many individuals expecting to achieve full functional recovery much sooner than actual surgical outcomes reported in the literature. ${ }^{5,6}$ In the months after TKR, as many as $11.8 \%$ of patients experience a fall, and up to $8 \%$ of patients are readmitted to the hospital. 7,8

Patients have reported having unrealistic expectations about the functional tasks they would be able to complete in the early postoperative phase with some individuals having significant disparities between their expectations and their perceived functional performance after surgery. ${ }^{6}$ Many patients are still unable to return to higher level functional tasks, such as gardening, by 6 months after surgery or tasks that require bending and stooping even after 1 year of recovery following TKR. ${ }^{9,10}$ Patients have also reported receiving conflicting information from health care providers, creating confusion regarding which instructions to follow at home. ${ }^{11,12}$ Additionally, the amount and intensity of pain experienced after knee replacement may exceed the discomfort patients expect to experience; some patients have reported having longer recovery times, sleep disturbances, and poor satisfaction due to unexpected levels of pain following joint replacement. ${ }^{11,13,14}$ Previous researchers discussing negative experiences following TKR demonstrated that patients may not be as prepared as they need to be to achieve optimal outcomes after discharge. Preparing for discharge after surgery begins with preoperative education to ready patients for the postoperative phase. Realistic expectations for recovery after TKR, knowledge about how to complete daily life tasks after surgery, strategies for preventing falls, and known factors related to hospital readmission should all be addressed through preoperative education as a resource for better preparing patients for return to home.

Little research is currently available in which patient or provider perceptions are explored regarding the adequacy of preoperative education for TKR. Scant literature is available regarding preoperative education for other diagnoses. Following an extensive literature review, only one study was found in which patient perceptions of preoperative education before cardiac surgery were explored, and another researcher studied patient perceptions regarding preoperative education for orthopedic surgery prior to reduction and internal fixation to repair fractures of the upper and lower extremities. ${ }^{15,16}$ While patients in both studies perceived that they received helpful information prior to surgery, areas identified as needing more education included expectations for the early postoperative phase. Montin et al. examined patients' perceptions of knowledge received preoperatively for total hip replacement (THR) and TKR using a questionnaire design. ${ }^{17}$ Montin et al. identified topics patients felt they received the most and least information about; however, a limitation of that study was it did not examine whether or not patients found the education to be adequate or areas they felt were missing. ${ }^{17}$ Ingaddotir et al. found that patients' knowledge expectations before TKR exceeded the knowledge gained through preoperative education, demonstrating a disparity between knowledge desired and knowledge obtained prior to surgery. ${ }^{18}$ Literature regarding provider perceptions about preoperative and postoperative education is very limited. In a graduate research project, Rae identified occupational therapists' perceptions about the ideal preoperative education program for individuals with planned THR surgery. ${ }^{19}$ Rae found discrepancies between the current program design for THR and the participants' perceptions regarding the optimal design for preoperative education with this population. ${ }^{19}$ Westby and Backman examined patient and health care providers' views of rehabilitation practices and outcomes after TKR and THR and found different but overlapping perceptions among these two groups. ${ }^{14}$ Patients found communication with providers to be inadequate while health professionals focused more on the inadequacy of communication between disciplines. Both patients and health care professionals expressed that preoperative education was crucial for providing clarification regarding expectations and empowering the clients to be an active participant in their recovery. ${ }^{14}$

(C) Internet Journal of Allied Health Sciences and Practice, 2018 
The current evidence regarding the efficacy of preoperative education presents conflicting results. Larger systematic reviews of randomized controlled trials have demonstrated that available research is not strong enough to support or contradict the use of preoperative education prior to joint replacement surgery. ${ }^{20-22}$ Individual researchers have found positive results of preoperative education, such as reduced anxiety prior to surgery, increased preparedness for surgery, decreased fall incidence, better pain control post-operatively, and reduced length of stay. ${ }^{23-27}$ However, other researchers have shown that preoperative education did not fully prepare patients for the expectations after surgery with many patients finding both preoperative and postoperative education to be insufficient. ${ }^{14,28,29}$ Some patients have reported receiving inconsistent or even contradictory information from different health care providers. 11,12,27 Previous researchers have reflected that patient education is not fully addressing the needs of TKR patients prior to discharge. The structure of preoperative education programs used nationally for patients prior to TKR surgery has not been identified previously, thus hospitals across the United States lack a common standard for this education to ensure best outcomes to promote safety, increased functional independence, and improved quality of life for discharge home.

Limited descriptions of educational content, delivery methods, and providers of preoperative education have been provided in individual research manuscripts and have also been presented in systematic reviews for the studies that met strict inclusion criteria to be incorporated in these reviews. ${ }^{20-22}$ Descriptions of programs currently found in systematic reviews or individual studies within the literature are not representative of preoperative education at a national level. A variety of health care staff may participate in preoperative education, such as nurses, physical therapists, occupational therapists, social workers/discharge planners among other providers, who have a role in delivering preoperative education. These providers along with the content and methods of educational delivery can vary significantly by program. An understanding of the current structure of preoperative education is needed as a basis to develop educational programs that better meet client needs to prepare them for discharge home. The objectives of this pilot survey study were to identify the content, providers and, delivery methods of preoperative education programs for patients undergoing TKR surgery.

\section{METHODS}

This pilot study was approved by the Institutional Review Board (IRB) at Eastern Kentucky University through expedited review in September 2016. All participants provided informed consent to participate in the study and were advised that participation was voluntary and could be stopped at any time.

\section{Research Design}

This research included an exploratory, descriptive design with an online pilot survey created by the investigators. The survey consisted of both closed-ended and open-ended question items and was conducted in fall 2016 and spring 2017. Survey research is an appropriate technique for presenting descriptive data about a topic that has not been thoroughly researched previously. ${ }^{30}$ This method also was used gathering information across a broader audience and geographic area in a more efficient manner than other methods of data collection, such as in-person interviews. ${ }^{30}$

\section{Recruitment.}

The population for this study included all health care providers who currently participate in preoperative education for persons undergoing TKR surgery. An exhaustive list of these providers was not available, which did not permit determining the total number of individuals who are included in this population. Because no comprehensive database exists that lists all providers or facilities that participate in preoperative education for TKR surgery, potential participants were recruited for the study by contacting providers from hospitals at geographic locations around the United States who provide preoperative education to this patient population. These hospitals were located through convenience sampling using contacts of the first author as well as an Internet search of hospitals that provide TKR and an education program prior to surgery. Hospitals that met these criteria were contacted throughout the study time period to identify an appropriate individual at the facility who provided preoperative education. Potential participants were contacted via telephone and email by the primary investigator. The main hospital number was used as the initial point of contact if a direct phone number or email address for preoperative education or total joint replacement were not available on the hospital's Web site. The primary investigator described the study in detail and asked if the individual would like to 
participate in the study. The primary investigator then emailed the informed consent letter and a link to the survey for study completion. Forty facilities were contacted for recruitment, and 15 individuals initially agreed to participate in the study. Through the informed consent letter, email cover letter, and telephone conversation with the primary investigator, participants were advised that participation was voluntary and could be stopped at any time. Completion of the survey was considered as providing informed consent for participation in the study.

\section{Data Collection:}

Methods and Instrumentation. Data were collected using an online survey. Participants were emailed a link to complete the survey through the Research Electronic Data Capture (REDCap), a secure system that supports Webbased data collection and analysis for research studies. ${ }^{31}$ This program can be accessed through a university affiliation. The survey included 16 questions total and took approximately 10 minutes to complete. See Appendix A for a copy of the survey questions. Survey items posed questions about demographic information, current program design, ideal program design, and feedback regarding the design of the survey itself. Demographic questions numbers 1 through 3 asked participants to identify their health care discipline, how long they had worked in their discipline, and their educational level. Current program design questions numbers 4 through 10 were focused on the following areas related to preoperative education: topics covered, providers involved, educational delivery methods, format, timing, number of sessions, and length of sessions. Questions numbers 11 through 13 regarding ideal educational programs asked participants to identify optimal design for these same areas as well as asking participants to identify any other needed alterations to their current program design. Open-ended questions numbers 14 through 16 at the end of the survey sought feedback regarding the survey design itself, including the following: any areas of confusion on the survey, feedback regarding additional survey questions or response items that participants felt should be added, and any additional feedback that participants wanted to add regarding the design of the survey. Survey questions were developed based on information available in the literature describing preoperative education as well as by reviewing 3 preoperative education programs in Lexington, Kentucky. The first author either attended a preoperative education class or met with the primary education provider for each of these 3 programs. Materials available from these programs, such as booklets and PowerPoint presentations, were also reviewed by the first author.

Data Analysis. Data were analyzed using the "Data Exports, Reports, and Stats" feature from REDCap. Descriptive statistics were used for data analysis to determine frequency and percentages for responses on closed-ended survey items. Responses on open-ended survey items were recorded and collated from individual survey responses.

\section{RESULTS}

\section{Participant Demographics}

Out of 15 providers who initially agreed to participate in the research from the 40 facilities that were contacted, 7 participants representing different facilities completed the pilot survey during the study timeframe. Two were nurses, 1 was a physical therapist, and 4 were occupational therapists. Three participants had between 2 and 5 years of experience in practice while 4 had more than 10 years of experience in their respective health care disciplines. One participant had completed an associate's degree, 2 had completed bachelor's degrees, 3 had completed master's degrees, and 1 participant had completed a clinical doctorate as the highest level of education.

\section{Current Program Design}

Additional Providers. The majority of participants reported having a preoperative education team that included nursing $(6 / 7 ; 85.7 \%)$, physical therapy $(6 / 7 ; 85.7 \%)$, and occupational therapy $(5 / 7 ; 71.4 \%)$ as additional providers involved in preoperative education at their facilities. Social work and case management were each reported by 2 participants $(2 / 7 ; 28.6 \%)$ to be included as part of the preoperative education team. Providers from dietetics were not involved in any of the preoperative programs in this study. Three participants reported other health care providers who included providers from pain management, hospital concierge services, and home health care.

Educational Topics. Participants educated patients on a wide variety of topics in preoperative classes at their facilities (see Table 1). All participants reported covering how to prepare for surgery, the expectations while in the hospital, adaptive equipment, self-care tasks, home safety, pain management, and exercise before and/or after surgery. The 
recovery process, functional mobility, home modifications, and precautions were also commonly included as educational topics. Anatomy of the knee joint and expected functional outcomes were covered in a little more than half of participants' programs. Topics less commonly included in preoperative education were instrumental activities of daily living (such as completing laundry and caring for the home), caregiver training, and when to resume normal activities at home. One participant $(1 / 7 ; 14.3 \%)$ reported an additional area covered during education related to the surgical procedure itself, and another responded that his or her program provided education on the need to increase protein and fiber intake along with preventing and treating constipation.

Table 1. Educational Topics Included in Preoperative Education

\begin{tabular}{lc}
\hline \multicolumn{1}{c}{$\begin{array}{c}\text { Educational } \\
\text { Topic }\end{array}$} & $\begin{array}{c}\text { Number of Participants } \\
\text { Frequency and Percent }\end{array}$ \\
\hline How to prepare for surgery & $7 / 7(100 \%)$ \\
Expectations while in the hospital & $7 / 7(100 \%)$ \\
The recovery process & $6 / 7(85.7 \%)$ \\
Self-care (such as dressing, bathing, toileting) & $7 / 7(100 \%)$ \\
Functional mobility (such as transfers) & $6 / 7(85.7 \%)$ \\
Instrumental activities of daily living (such as completing laundry & $3 / 7(42.9 \%)$ \\
and caring for the home) & \\
Adaptive equipment & $7 / 7(100 \%)$ \\
Home modifications & $5 / 7(71.4 \%)$ \\
Home safety & $7 / 7(100 \%)$ \\
Managing pain & $7 / 7(100 \%)$ \\
Caregiver training & $3 / 7(42.9 \%)$ \\
Exercise before and/or after surgery & $7 / 7(100 \%)$ \\
Precautions & $6 / 7(85.7 \%)$ \\
Anatomy of the knee joint & $4 / 7(57.1 \%)$ \\
When to resume normal activities at home & $3 / 7(42.9 \%)$ \\
Expected functional outcomes & $4 / 7(57.1 \%)$ \\
Other & $2 / 7(28.6 \%)$ \\
\hline
\end{tabular}

Educational Delivery Methods. All participants reported that both verbal and written instruction were used to educate TKR patients prior to surgery. Demonstration was used by $6(6 / 7 ; 85.7 \%)$ participants while video and online education were both used by $2 / 7(28.6 \%)$ participants. Workbooks were not used by any of the participants in this study. Three participants $(3 / 7 ; 42.9 \%)$ indicated that other methods were used for presurgical education. Two participants $(2 / 7$; $28.6 \%$ ) stated that a PowerPoint presentation was used as part of the education process. The third response included using the teach-back method or return demonstration as an educational technique.

Preoperative Education Program Structure. Most participants (4/7; $57.1 \%)$ reported that patients typically attended preoperative education 2 weeks prior to surgery. The timeframe in which patients usually attended preoperative education at other facilities varied from less than 1 week $(1 / 7 ; 14.3 \%)$, to 3 weeks $(1 / 7 ; 14.3 \%)$ and up to 4 weeks $(1 / 7$; $14.3 \%)$ before undergoing TKR. Most participants $(5 / 7 ; 71.4 \%)$ reported that their facility used a group format to provide presurgical education. One participant $(1 / 7 ; 14.3 \%)$ responded that the facility used both an individual and group education format, and another participant $(1 / 7 ; 14.3 \%)$ reported that preoperative education was provided only on an 
individual basis. Five participants $(5 / 7 ; 71.4 \%)$ reported that patients attended only 1 session prior to TKR surgery. One participant $(1 / 7 ; 14.3 \%)$ worked at a facility in which patients attended 2 sessions before surgery, and the remaining participant $(1 / 7 ; 14.3 \%)$ worked at a facility in which patients attended 5 or more preoperative education sessions. Length of preoperative education sessions varied, but no sessions were shorter than 1 hour or longer than 2.5 hours. Participants reported sessions lasting 1 hour to less than 1.5 hours (4/7; $57.1 \%), 1.5$ hours to less than 2 hours (1/7; $14.3 \%)$, and 2 hours to less than 2.5 hours $(2 / 7 ; 28.6 \%)$.

\section{Optimal Program Design}

Recommended Additional Educational Topics. Five of the 7 participants reported additional topics they believed would be beneficial to be included in preoperative education, and 2 out of the 7 participants reported that there were no educational topics they would recommend as additions to their current educational programs (see Table 2). One participant $(1 / 7 ; 14.3 \%)$ felt that functional mobility should be added to the educational program at his or her facility, another $(1 / 7 ; 14.3 \%)$ wanted to include education about the anatomy of the knee joint, and 1 participant $(1 / 7 ; 14.3 \%)$ also wanted to teach patients about home modification before surgery. Two participants $(2 / 7 ; 28.6 \%)$ would prefer that instrumental activities of daily living (such as completing laundry and caring for the home) be added as a part of preoperative education. Two participants $(2 / 7 ; 28.6 \%)$ also recommended both caregiver training and education about expected functional outcomes of TKR surgery.

Table 2. Additional Educational Topics Recommended for Preoperative Education

\begin{tabular}{lc}
\hline \multicolumn{1}{c}{ Educational Topic } & $\begin{array}{c}\text { Number of Participants } \\
\text { Frequency and Percent }\end{array}$ \\
\hline How to prepare for surgery & $0 / 7(0 \%)$ \\
Expectations while in the hospital & $0 / 7(0 \%)$ \\
The recovery process & $0 / 7(0 \%)$ \\
Self-care (such as dressing, bathing, toileting) & $0 / 7(0 \%)$ \\
Functional mobility (such as transfers) & $1 / 7(14.3 \%)$ \\
Instrumental activities of daily living (such as completing laundry & $2 / 7(28.6 \%)$ \\
and caring for the home) & $0 / 7(0 \%)$ \\
Adaptive equipment & $1 / 7(14.3 \%)$ \\
Home modifications & $0 / 7(0 \%)$ \\
Home safety & $0 / 7(0 \%)$ \\
Managing pain & $2 / 7(28.6 \%)$ \\
Caregiver training & $0 / 7(0 \%)$ \\
Exercise before and/or after surgery & $0 / 7(0 \%)$ \\
Precautions & $1 / 7(14.3 \%)$ \\
Anatomy of the knee joint & $0 / 7(0 \%)$ \\
When to resume normal activities at home & $2 / 7(28.6 \%)$ \\
Expected functional outcomes & $0 / 7(0 \%)$ \\
Other & $2 / 7(28.6 \%)$ \\
None &
\end{tabular}

Recommended Additional Health Care Providers. When asked which additional providers participants felt would be beneficial for their patients, the majority of participants $(4 / 7 ; 57.1 \%)$ indicated that they did not have recommendations 
to add any additional providers to the current preoperative education program at their facility. Three participants (3/7; $42.9 \%$ ) would like to have case management involved in preoperative education, and 1 participant $(1 / 7 ; 14.3 \%)$ would like to have social work become involved with the program. One participant $(1 / 7 ; 14.3 \%)$ indicated that he or she would like to see the physician assistant and/or surgeon become involved in providing preoperative education before TKR surgery.

Other Recommended Alterations to Preoperative Education. Participants were asked to provide any other additions or alterations they felt were needed to their current preoperative education program to best meet the needs of their patients. Three participants $(3 / 7 ; 42.9 \%)$ provided responses to this question item. One participant $(1 / 7 ; 14.3 \%)$ wanted to have the preoperative education program expanded to include all physicians at his or her facility because currently only patients from two surgeons utilize the program. Another participant recommended that the combined orthopedic class be completed separately for TKR patients and those who undergo THR. The remaining participant felt that it would be valuable to add a video of a TKR patient completing active and passive range of motion exercises with a physical therapist to reinforce the amount of effort required for patients to achieve optimal outcomes after surgery.

\section{DISCUSSION}

With this pilot study, we found that current programs for preoperative education included a variety of educational topics with some areas being covered less frequently among programs than others. Other aspects of program design varied as well, such as when preoperative education was provided prior to surgery. Several participants had suggestions to improve the current structure of their preoperative education programs related to topics covered, providers involved, and other recommendations. Participant responses also provided insight to improve the design of the survey for future implementation on a larger scale.

\section{Current Preoperative Education Program Design}

Health Care Providers. Nursing and physical therapy have commonly been reported in the literature in systematic reviews that have described providers included in preoperative education, which is similar to the results of this pilot research. In these same studies, researchers have not found occupational therapy to be commonly involved in preoperative education after total joint replacement surgery, which contradicts the findings of this study. ${ }^{20-22}$ Louw et al. listed the top 10 providers involved in preoperative education for the studies included in their systematic review, and occupational therapy was not among those disciplines listed. ${ }^{20}$ The discrepancy between this pilot study and the literature could be due to participant demographics, which included 4 out of 7 participants who were occupational therapists as well as the use of convenience sampling methods. Occupational therapy may be beneficial to include in preoperative education prior to TKR surgery to address areas related to self-care as well as higher level functional tasks following surgery. ${ }^{32}$

Educational Topics. Similar to this study, common topics covered in preoperative education that have been reported in previous research include preparing for surgery, expectations after surgery, the recovery process, self-care, functional mobility, adaptive equipment, managing pain, precautions, safety at home, and exercise.20-22 Other programs have covered additional aspects, such as finances related to surgery, advice from previous patients who underwent joint replacement, and the roles of medical support staff. ${ }^{33}$ Including an "other" option for participants on this pilot survey and on the revised survey for future implementation will capture additional topics covered at individual facilities. While pain management was addressed by all participants in this study, previous researchers have demonstrated that this education may be inadequate to prepare patients for the frequency and intensity of pain that they experience after

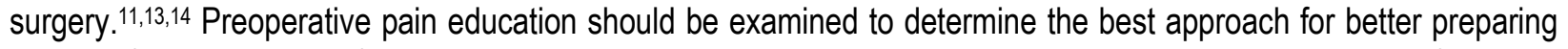
patients for this aspect of the recovery process, and patients should be provided with more realistic information regarding the discomfort they may experience after surgery.

Patients need to be educated about when most individuals resume various activities following surgery as patients often have unrealistic expectations regarding their functional outcomes and the timeframe in which they can return to their regular activities after surgery. ${ }^{15,16}$ Caregiver training is not a common education topic either; however, some patients have identified that including their support system during preoperative education increases their confidence for 
discharge following surgery. ${ }^{12,13}$ Patients should be provided information regarding adaptive equipment or techniques that may assist daily activities post surgery. Surprisingly, individual differences among TKR patients, such as related to medical history, were not identified as being addressed in the pilot study or as a common theme in the literature. For example, a systematic review in which the content of preoperative education addressing post-operative pain for THR and TKR was explored did not identify differentiating pain between patients with rheumatoid arthritis and osteoarthritis in preoperative education. ${ }^{20}$

Educational Delivery Methods. Verbal and written instruction were the most commonly reported methods for delivering education before surgery in the literature as well as in this pilot study. ${ }^{20-22}$ Some researchers have also examined the impact of video education combined with verbal instruction versus verbal instruction alone, but did not find significant functional differences in favor of the addition of video. ${ }^{32,34}$ Heikkinen et al. compared Internet-based education alone to face-to-face education and found some evidence that Web-based education provided a higher level of knowledge preoperatively than face-to-face education with staff. ${ }^{33}$ There is not a clear best method for educational delivery provided in the literature.

Timing and Format of Preoperative Education. Similar to results in this pilot study, the timing of preoperative education varies widely in the literature. Previous researchers have reported preoperative education occurring as much as 6 weeks before planned total joint replacement with most scheduled within 4 weeks prior to the procedure and as late as the evening before surgery.20-22,35,36 The timing of education prior to TKR surgery has not been studied specifically in the literature and warrants examination. Providing education too early could mean that patients forget important information by the time of their surgery while providing this education too late can mean that patients do not have time to absorb new information that is important for their recovery. Patients may also not have time to ask questions that could better prepare them for surgery in the postoperative phase.

The majority of participants in this pilot study reported that presurgical education was provided in a group format. However, most research reported in the literature showed that education sessions were more evenly divided between one-on-one verbal education and group sessions with the least common format using methods that did not include face-to-face contact with a health care provider. ${ }^{20-22,24}$ Individual versus group format for educational delivery has not been studied extensively in the literature and is another area that should be examined to determine the effectiveness of delivering educational content to a single patient compared with providing this same information to multiple patients at a time.

Length and Number of Preoperative Education Sessions. Length of preoperative education sessions varied widely in the literature from 12 minutes (video format only) to half a day, compared with a range of 1 hour to 2.5 hours in this study. ${ }^{20-22}$ Attending one educational session prior to surgery was commonly reported in the literature. With this pilot research, few programs included more than 1 preoperative education session; however, some researchers examining preoperative education included multiple presurgical exercise sessions as part of the intervention. ${ }^{20-22}$

\section{LIMITATIONS}

One limitation of this study was a small sample size. While 40 facilities were contacted and 15 providers initially agreed to participate, only 7 participants completed the survey in the study timeframe. In other health care research reported in the literature, there have been small sample sizes from 6 to 10 participants for the initial pilot study prior to administration of the survey on a much larger population. ${ }^{37,38}$ The recruitment method for this study was another limitation as convenience sampling used for this pilot research may not provide the substantial number of participants required for a large-scale study in the future. The study also included a disproportionate ratio of health care providers who participated in the survey with 4 participants being occupational therapists. Additionally, the geographic location of participants was not recorded on the surveys. While participants from across the United States were recruited for the study, surveys were completed anonymously, and it is not possible to determine the geographic representation of the study sample. A question item will be added to the survey for future research to obtain geographic location by state for participants. Another limitation was evident based on responses from participants on the question item related to additional providers who were involved in preoperative education. For example, 4 out of 7 occupational therapists 
completed the survey, but 5 participants indicated occupational therapy as additional providers involved in preoperative education at their facilities. The suggestion of this finding is that either more than one occupational therapist was involved in preoperative education at a portion of these facilities, or some participants included themselves in their response to this question. Altering this question for the final survey should ensure that participants have a similar understanding of this item when they respond to this question.

\section{SUMMARY}

With this pilot study, we begin to describe the nature of current preoperative education programs for TKR surgery in the United States as well as to pilot the survey and mode of survey delivery for future research. Seven participants completed the survey and provided insight regarding how program structures varied across health care sites. Changes will be made for future implementation of the survey based on analysis of responses on the survey items. There are currently limited descriptions of preoperative education programs provided in the literature, and these program designs vary across settings. With this pilot research, we provided a beginning framework for establishing usual practice, which can later be used as a basis for determining best practice for preoperative education prior to TKR surgery. A large, national survey is needed to fully describe the content, providers, and delivery methods of preoperative education for TKR patients across the United States using a representative sample. Results of this larger survey could be used to inform future research to examine the effectiveness of various program designs once their structures are fully known in order to develop programs that will support better postoperative outcomes. Additional future research could examine other related areas, such as patient understanding, perceptions, and expectations regarding preoperative education for TKR surgery.

DECLARATION OF INTEREST: The authors report no declarations of interest. The authors alone are responsible for the content and writing of this paper. 


\section{References}

1. Deshpande BR, Katz JN, Solomon DH, Yelin EH, Hunter DJ, et al. Number of persons with symptomatic knee osteoarthritis in the US: Impact of race and ethnicity, age, sex, and obesity. Arthritis Care Res. 2016;68(12):1743-50. doi:10.1002/acr.22897 [PMID: 27014966]

2. Beyond Surgery: The Full Impact of Knee Replacement. American Academy of Orthopedic Surgeons [Internet]. (2014). The Association; 2017. Available at: http://www.anationinmotion.org/value/knee/. Accessed June 22, 2017.

3. Skou ST, Roos EM, Laursen MB, Rathleff MS, Arendt-Nielsen L, et al. A randomized, controlled trial of total knee replacement. N Engl J Med. 2015;373:1597-606. doi:10.1056/NEJMoa1505467 [PMID: 26886535]

4. Shan L, Shan B, Suzuki A, Nouh F, Saxena A. Intermediate and long-term quality of life after total knee replacement: A systematic review and meta-analysis. J Bone Joint Surg Am. 2015;97(2):156-68.

doi:10.2106/JBJS.M.00372 [PMID: 25609443]de Achaval S, Kallen MA, Amick B, Landon G, Siff S, et al. Patients' expectations about total knee arthroplasty outcomes. Health Expect. 2016;19(2):299-308. doi:10.1111/hex.12350 [PMID: 25684135]

5. Levinger P, Diamond N, Menz H, Wee E, Margelis S, et al. Development and validation of a questionnaire assessing discrepancy between patients' pre-surgery expectations and abilities and postsurgical outcomes following knee replacement surgery. Knee Surg Sports Traumatol Arthrosc. 2016;24(10):3359-68. doi:10.1007/s00167-014-3432-4 [PMID: 25423872]

6. Swinkels A, Newman JH, Allain TJ. A prospective observation study of falling before and after total knee replacement surgery. Age Ageing. 2009;38:175-81. doi: 10.1093/ageing/afn229 [PMID: 19029071]

7. Schairer W, Vail T, Bozic K. What are the rates and causes of hospital readmission after total knee arthroplasty? Clin Orthop Relat Res. 2014;472(1):181-7. doi:10.1007/s11999-013-3030-7 [PMID: 23645339]

8. Hamel MB, Toth M, Legedza A, Rosen MP. Joint replacement surgery in elderly patients with severe osteoarthritis of the hip and knee: Decision making, postoperative recovery, and clinical outcomes. Arch Intern Med. 2008;168(13):1430-40. doi: 10.1001/archinte.168.13.1430 [PMID: 18625924]

9. White L, Stockwell T, Hartnell N, Hennessy M, Mullan J. Factors preventing kneeling in a group of preeducated patients post total knee arthroplasty. J Orthop Traumatol. 2016:17(4):333-8. doi:10.1007/s10195-016-0411-1 [PMID: 27234004]

10. Goldsmith LJ, Suryaprakash N, Randall E, Shum J, MacDonald V, et al. The importance of informational, clinical and personal support in patient experience with total knee replacement: $A$ qualitative investigation. BMC Musculoskelet Disord. 2017;18(127). doi:10.1186/s12891-017-1474-8 [PMID: 28340610]

11. Specht $K$, Kjaersgaard-Andersen $P$, Pedersen $B D$. Patient experience in fast-track hip and knee arthroplasty - a qualitative study. J Clin Nurs. 2016;25(5-6):836-45. doi:10.1111/jocn.13121 [PMID: 26708610]

12. Causey-Upton R, Howell DM. Patient experiences when preparing for discharge home after total knee replacement. Internet J Allied Health Sci Pract. 2017;15(1). http://nsuworks.nova.edu/ijahsp/vol15/iss1/5

13. Westby MD, Backman CL. Patient and health professional views on rehabilitation practices and outcomes following total hip and knee arthroplasty for osteoarthritis: A focus group study. BMC Health Serv Res. 2010;10:119. doi: 10.1186/1472-6963-10- 119 [PMID: 20459834]

14. Chetty C, Ehlers VJ. Orthopaedic patients' perceptions about their pre-operative information. Curationis. 2009;32(4):55-60. doi: 10.4102/curationis.v32i4.992

15. O'Brien L, McKeough $C$, Abbasi R. Pre-surgery education for elective cardiac surgery patients: A survey from the patient's perspective. Aust Occup Ther J. 2013;60(6):404-9. doi: 10.1111/1440-1630.12068 [PMID: 24299479] 
16. Montin L, Johansson K, Kettunen J, Katajisto J, Leino-Kilpi H. Total joint arthroplasty patients' perception of received knowledge of care. Orthop Nurs. 2010;29(4):246-53. doi: 10.1097/NOR.0b013e3181e51868 [PMID: 20664463]

17. Ingaddotir B, Stark AJ, Leiono-Kilpi H, Sigurdardottir AK, Valkeapaa K, et al. The fulfilment of knowledge expectations during the perioperative period of patients undergoing knee arthroplasty- a Nordic perspective. J Clin Nurs. 2014;23(19-20):2896-908. doi: 10.1111/jocn.12552 [PMID: 24476393]

18. Rae S. Pre-surgery education with clients undergoing total hip replacements (Unpublished master's theses). University of Puget Sound, Tacoma, WA; 2011. Available at http://soundideas.pugetsound.edu/ms occ therapy/25/. Accessed June 22, 2017.

19. Louw A, Diender I, Butler DS, Puentedura EJ. Preoperative education addressing postoperative pain in total joint arthroplasty: Review of content and educational delivery methods. Physiother Theory Pract. 2013;29(3):175-94. doi: 10.3109/09593985.2012.727527 [PMID: 23035767]

20. McDonald S, Hetrick S, Green S. Pre-operative education for hip or knee replacement. Cochrane Database Syst Rev. 2004;1. doi:10.1002/14651858.CD003526.pub2 [PMID: 14974019]

21. McDonald S, Page MJ, Beringer K, Wasiak J, Sprowson A. Preoperative education for hip or kneereplacement. Cochrane Database Syst Rev. 2014;13(5). doi: 10.1002/14651858.CD003526.pub3 [PMID: 24820247]

22. Chen S-R, Chen C-S, Lin P-C. The effect of educational intervention on the pain and rehabilitation performance of patients who undergo a total knee replacement. J Clin Nurs. 2014;23(1-2):279-87. doi: 10.1111/jocn.12466 [PMID: 24313941]

23. Clarke HD, Timm VL, Goldberg BR, Hattrup SJ. Preoperative patient education reduces in-hospital fall after total knee arthroplasty. Clin Orthop Relat Res. 2012;470(1):244-9. doi: 10.1007/s11999-011-19516 [PMID: 21691907]

24. Jones S, Alnaib M, Wilkinson M, St Clair Gibson A, Kader D. Pre-operative patient education reduces length of stay after knee joint arthroplasty. Ann R Coll Surg of Engl. 2011;93(1):71-5. doi: 10.1308/003588410X12771863936765 [PMID: 21418755]

25. Kearney M, Jennrich MK, Lyons S, Robinson R, Berger B. Effects of preoperative education outcomes after joint replacement surgery. Orthop Nurs. 2011;30(6):391-6. doi: 10.1097/NOR.0b013e31823710ea [PMID: 22124192]

26. Spalding J. Reducing anxiety by pre-operative education: Making the future familiar. Occup Ther Int. 2003;10(4):278-93. [PMID: 146457541]

27. Fielden JM, Scott S, Horne JG. An investigation of patient satisfaction following discharge after total hip replacement surgery. Orthop Nurs. 2003;22(6):429-36. [PMID: 14705473]

28. Jacobson AF, Myerscough RP, DeLambo K, Fleming E, Huddleston AM, et al. Patients' experiences on total knee replacement: A qualitative study sheds light on pre- and postoperative experiences. Am J Nurs. 2008;108(5):54-63. doi: 10.1097/01.NAJ.0000318000.62786.fb [PMID: 18434802]

29. Fowler FJ. Survey Research Methods, 5e. Thousand Oaks, CA: Sage Publications, Inc.;2014

30. Harris PA, Taylor R, Thielke R, Payne J, Gonzalez N, et al. (2009). Research electronic data capture (REDCap) - A metadata-driven methodology and workflow process for providing translational research informatics support. J Biomed Inform. 2009;42(2):377-81. doi: 10.1016/j.jbi.2008.08.010 [PMID: 18929686]

31. Rastogi R, Davis AM, Chesworth BM. A cross-sectional look at patient concerns in the first six weeks following total knee arthroplasty. Health Qual Life Outcomes. 2007;5:48. doi:10.1186/1477-7525-5-48 [PMID: 17678532]

32. Heikkinen K, Helena L-K, Nummela T, Kaljonen A, Sanna S. A comparison of two educational interventions for the cognitive empowerment of ambulatory orthopedic surgery patients. Patient Educ Couns. 2008;73(2):272-9. doi: 10.1016/j.pec.2008.06.015 [PMID: 18678461] 
33. Leal-Blanquet J, Alentorn-Geli E, Gines-Cespedosa A, Martinez-Diaz S, Caceres E, et al. Effects of an educational audiovisual videodisc on patients' pre-operative expectations with total knee arthroplasty: a prospective randomized comparative study. Knee Surg Sports Traumatol Arthrosc. 2013;21(11):2595602. doi: 10.1007/s00167-012-2158-4 [PMID: 22878435]

34. Williams G. Causes of delayed discharge following joint replacement surgery. Int J Ther Rehabil. 2010;17(1):34-9. https://doi.org/10.12968/ijtr.2010.17.1.45991

35. Sjoling M, Nordahl G, Olofsson N, Asplund K. The impact of preoperative information on state anxiety, postoperative pain and satisfaction with pain management. Patient Educ and Couns. 2003;51(2):169-76. [PMID: 14572947]

36. Csajka C, Jaquel A, Winterfeld U, Meyer Y, Einarson A, et al. Risk perception by healthcare professionals related to drug use during pregnancy: A Swiss survey. Swiss Med Wkly. 2014;144. doi:10.4414/smw.2014.13936 [PMID: 24610065]

37. Hawkins SC, Osborne A, Egbeare D, Williamson J, Lambert AW. A positive change in surgical attitudes towards online workplace-based assessment. Ann R Coll Surg Engl (Suppl). 2014;96:e6-9. https://doi.org/10.1308/147363514X14042954769636 